\title{
Incipient sliding of adhesive contacts
}

\author{
Francesc PÉREZ-RÀFOLS ${ }^{1, *}$, Lucia NICOLA ${ }^{1,2}$ \\ ${ }^{1}$ Department of Industrial Engineering, University of Padova, Padua I-35131, Italy \\ ${ }^{2}$ Department of Material Science and Engineering, Delft University of Technology, Delft $2628 \mathrm{CN}$, the Netherlands \\ Received: 11 June 2021 / Revised: 16 July 2021 / Accepted: 03 August 2021 \\ (C) The author(s) 2021.
}

\begin{abstract}
A model is proposed herein to investigate the incipient sliding of contacts in the presence of both friction and adhesion, where the interfacial response is modeled based on traction-separation laws. A Maugis-like parameter is defined to characterize the response in the tangential direction. Subsequently, the model is used to investigate the contact between a smooth cylinder and a flat body, where adhesion-friction interactions are strong. A range of behaviors are observed when a tangential displacement is imposed: When the parameter is low, the contact pressure exhibits a relatively constant profile; when it is high, a pressure spike is observed at the edge of the contact. This difference is caused by a significant interface compliance in the former case, which limits the amount of slip. The results for the mid-range values of the Maugis-like parameter can qualitatively replicate various experiments performed using polydimethylsiloxane (PDMS) balls.
\end{abstract}

Keywords: incipient sliding; adhesion; traction-separation laws

\section{Introduction}

Friction in the presence of adhesion is an important issue in tribology. A comprehensive understanding of this problem is crucial to realize improvements in various applications, such as rubber adhesion in tireasphalt contact, adhesion in microelectromechanical systems (MEMS) or cells, interaction between nanoparticles [1], positioning methods, and reusable bio-inspired adhesives that can be removed without residue [2]. It is clear that adhesion and friction are correlated: Adhesion serves as a source of friction, while it decreases under friction force. The effect of this interaction on the contact area, total friction force, and contact pressure profile is yet to be elucidated.

The aim of this study is to investigate the evolution of the contact area, total friction force, and pressure distribution when a surface is tangentially loaded and the relative amount of adhesion, and friction are varied. In this study, an adhesive elastic smooth cylinder placed in contact with a rigid substrate was modeled, where the load applied tangentially was increased until gross slip was achieved. We performed this numerical study because experimental results in terms of the contact area and load are available for similar systems. In fact, various groups [2-7] have investigated the contact response of a soft polydimethylsiloxane (PDMS) ball on a rigid lens. Owing to significant adhesion on large areas, the contact area evolution can be measured and correlated with the frictional force. The behavior of the contact can be summarized as follows: As the tangential load increases from a value of zero, the contact area decreases through peeling, owing to the decrease in the adhesive interaction; additionally, it transforms from an initial circular shape to an elliptical or irregular shape [6, 7]. At this stage, the relationship between the contact area and tangential load follows a power law with an exponent of approximately 2 $[2,5,6]$. Eventually, sticking is no longer possible, and either contact loss or steady sliding occurs, depending on whether the normal load applied induces tension or compression [7]. A sticking-to-sliding transition may

* Corresponding author: Francesc PÉREZ-RÀFOLS, E-mail: francisco.perezrafols@unipd.it 


\begin{tabular}{|c|c|c|c|}
\hline \multicolumn{4}{|c|}{ Nomenclature } \\
\hline \multicolumn{2}{|c|}{ Dimensional variable } & \multicolumn{2}{|c|}{ Non-dimensional variable } \\
\hline$\delta$ & Characteristic length of adhesion (m) & $\lambda_{\mathrm{n}}$ & Maugis parameter \\
\hline$p$ & Pressure $(\mathrm{Pa})$ & $\lambda_{\mathrm{t}}$ & Maugis-like parameter in the tangentia \\
\hline$u$ & Displacement (m) & & direction \\
\hline$\phi$ & Work of adhesion $(\mathrm{N} / \mathrm{m})$ & $\tilde{G}_{i j}$ & Dimensionless Green's functions \\
\hline$\sigma_{\max }$ & $\begin{array}{l}\text { Maximum interface stress in normal } \\
\text { direction }(\mathrm{Pa})\end{array}$ & $\begin{array}{l}G \\
P\end{array}$ & $\begin{array}{l}\text { Gap between surfaces } \\
\text { Contact pressure }\end{array}$ \\
\hline$\tau_{\text {fit }}$ & $\begin{array}{l}\text { Fitting for the tangential stress at onset } \\
\text { of sliding }(\mathrm{Pa})\end{array}$ & $\begin{array}{l}U \\
W\end{array}$ & $\begin{array}{l}\text { Deformation at contact surface } \\
\text { Load }\end{array}$ \\
\hline$\tau_{\max }$ & $\begin{array}{l}\text { Maximum interface stress in tangential } \\
\text { direction }(\mathrm{Pa})\end{array}$ & $\begin{array}{l}X \\
\text { Superscript }\end{array}$ & Coordinate in tangential direction \\
\hline$\tau_{\infty}$ & Tangential stress at infinite separation $(\mathrm{Pa})$ & - & Point at which onset of sliding occurs \\
\hline $\boldsymbol{G}$ & Green's functions & S & Contact surface \\
\hline $\begin{array}{l}a_{\mathrm{r}} \\
E\end{array}$ & $\begin{array}{l}\text { Contact area }(\mathrm{m}) \\
\text { Elastic modulus }(\mathrm{Pa})\end{array}$ & Subscript & \\
\hline$E^{*}$ & Equivalent elastic modulus (Pa) & app & Applied \\
\hline$g$ & Gap between surfaces (m) & $\mathrm{dmp}$ & Damping \\
\hline$H_{1}$ & Height of the elastic block (m) & el & Elastic \\
\hline $\mathrm{H}_{2}$ & Height of the rigid block (m) & int & Interface \\
\hline$L$ & Length of periodic cell (m) & $\mathrm{n}$ & Normal direction \\
\hline$R$ & Radius of cylinder $(\mathrm{m})$ & $\mathrm{r}$ & Reference parameter used for scaling \\
\hline$w$ & Load between the two bodies $(\mathrm{N} / \mathrm{m})$ & total & Total \\
\hline$x$ & Coordinate in tangential direction (m) & $\mathrm{t}$ & Tangential direction \\
\hline
\end{tabular}

occur smoothly [2, 5] or via mechanical instability [3]. In the former case, steady sliding is typically preceded by a slight decrease in the measured tangential force $[3,5]$ and contact area [2].

Herein, we present a model that enables the relationship between friction and adhesion to be investigated; we verified the model by demonstrating that it can qualitatively reproduce experimental observations of strong and weak adhesion. Additionally, we provide some insights into the effect of adhesionfriction interactions on sliding by providing details that cannot be easily measured experimentally or calculated analytically, such as the tangential pressure profiles before and after the onset of slip. The tangential pressure distribution has only been measured experimentally for cases in which adhesion is not relevant $[8,9]$. In these cases, the tangential pressure is particularly high near the edges, where it soon reaches the highest value allowed. Hence local slip starts at the edges. Subsequently, the slip zone expands from the edges toward the center of the ball. The stick zone disappears gradually, and gross sliding commences. The initiation of gross sliding is a smooth process instead of an unstable one, as observed in some cases where adhesion is present [3]. However, we demonstrate that this does not necessarily apply when adhesion is significant. In particular, we allow the tangential stiffness to vary independently of the normal stiffness to determine its effect on the contact in general and the tangential pressure profile in particular.

The withdrawal of adhesive balls has been addressed analytically by several authors using models based on linear elastic fracture mechanics (LEFM) [4, 10-17]. These authors successfully replicated most experimental observations in which adhesion is strong, including 
the power law decay of the contact area with increasing tangential load, and its shape transformation from circular to elliptical [13]. Hence, we present a comparison between our model and these models. Although these models are effective, they are applicable only to the ball-on-flat problem, whereas the numerical model presented herein can be applied to any geometry.

Recently, by applying different local friction laws, Mergel et al. [2, 18] successfully developed a twodimensional finite element model (FEM) that can describe the reduction in adhesion through material expansion at the surface. In particular, an experimental trend showing a decrease in the contact area was replicated. However, their model predicts that this decay will vanish if the material is incompressible. In our opinion, this is problematic because the polymers used in most of the aforementioned experimental studies are incompressible.

In our model, the behavior of the interface is governed by traction-separation laws that describe the interaction between adhesion and friction. Specifically, the laws that we used are based on the cohesive zone model proposed by McGarry et al. [19]. These laws explicitly couple normal and tangential tractions; as such, the adhesive traction decreases as the tangential gap increases. This guarantees a decrease in the contact area provided that the adhesion is relevant. The manner in which this decrease occurs will be investigated in this study. These traction-separation laws predict that the contact pressure approaches zero rapidly as the tangential gap increases. This implies that sliding occurs at zero normal and zero tangential pressures, which contradicts with observations. In Ref. [20], this was addressed by allowing each grid point on a surface to interact with all grid points in the counter-surface based on the same traction-separation law. However, it was observed that the response was grid-size dependent, and that friction vanished when an extremely fine discretization was used. As this is not an acceptable feature, we modified the model presented in Ref. [21] proposing new tractionseparation laws, which were inspired by those presented in Ref. [19], and which can account for sliding at non-zero pressures.

Similar to Salehani et al. [21], we modelled the elastic response of the bodies using a Green's function approach [22]. The primary advantage of this approach compared with FEM is that meshing is required only at the contact surfaces. Although the simulations performed in this study did not incur a high cost, a fast computation of the fields is beneficial for investigating rough surfaces in future studies. The proposed model, which adopts the modified tractionseparation laws, can capture the experimental observations described above provided that a certain compliance is allowed at the interface. This compliance allows the interface to deform before the onset of slip and mimics interfacial bonding. It is controlled by a Maugis-like parameter that correlates the magnitude of the deformations of the interface with those of the body. The results obtained using a sufficiently small value of the Maugis-like parameter can replicate the experimental results presented in Refs. [2-6].

\section{Modeling approach}

The problem considered is presented in Fig. 1, where two linear elastic isotropic bodies are brought into contact with each other. One is flat and much more rigid than the other, which is incompressible and exhibits has the shape of a smooth cylinder with radius $R$. Nonetheless, we assume that the contact width is sufficiently small compared with $R$, and that the height of the bodies is sufficiently large such that the bodies can be approximated as half-spaces. Hence, the response of the system is independent of the manner by which the elastic modulus $E$ and the shape of the surfaces are partitioned. The flat body was fixed at the bottom in both the normal and tangential directions. Subsequently, loading was imposed by applying displacements $u_{\text {app }_{\mathrm{n}}}$ and $u_{\mathrm{app}_{\mathrm{t}}}$ at the top of the cylinder, where the subscripts $n$ and $t$ indicate the normal and

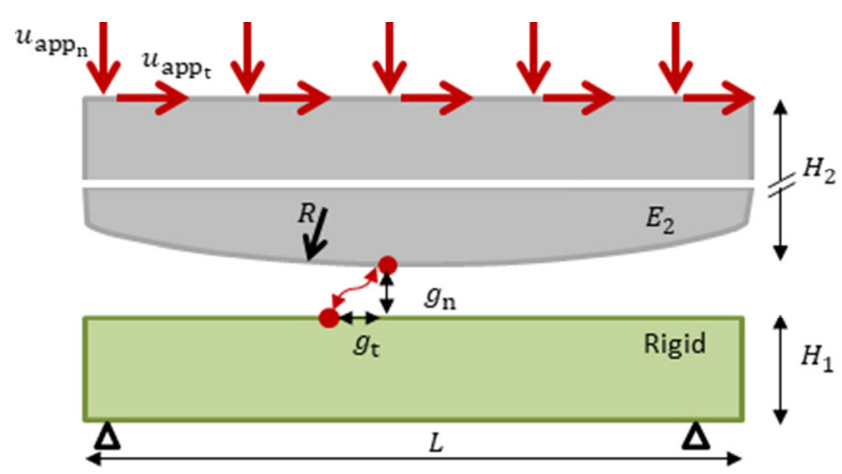

Fig. 1 Schematic representation of the problem considered in this study. 
tangential directions, respectively. The two bodies have a finite height $H$. When the two bodies are sufficiently close to each other, an interaction pressure $p_{\text {int }}$ emerges at the contact surface. This pressure is controlled by a local traction-separation law, which accounts for repulsive forces, adhesion, and friction, as will be described in Section 2.1.

The method presented in Ref. [21], which is based on Green's function approach presented in Ref. [22], was used in this study to compute the tractions and deformations at the interface. The assumptions allowed Green's functions to be defined based on the relationship between the elastic recovery force at the interface and the deformations of the elastic cylindrical body at the contact surface $\boldsymbol{u}^{\mathrm{s}}$ and top surface $\boldsymbol{u}_{\text {app }}$. Therefore, for this body, $p_{\mathrm{el}}=\boldsymbol{G} \boldsymbol{u}$, where $\boldsymbol{u}=\left[u_{\mathrm{n}}^{\mathrm{s}}, u_{\mathrm{t}}^{\mathrm{s}}, u_{\mathrm{app}_{\mathrm{n}}}, u_{\mathrm{app}_{\mathrm{t}}}\right]$, and $G$ is a matrix of Green's functions, as provided in Ref. [21]; Green's functions are obtained in the Fourier space. The total pressure at the interface is expressed as

$$
p_{\text {total }}=p_{\text {int }}+p_{\text {el }}+p_{\text {dmp }}
$$

where $p_{\mathrm{dmp}}$ is a damping pressure added to enhance the convergence and vanishes when the solution is obtained. In the same manner, as in Ref. [22], we used $p_{\text {dmp }}$ in a Varlet integration scheme to update the displacement of the discretization nodes at the surface until a stable position was achieved. It is noteworthy that, owing to the use of Fourier transforms, periodic boundary conditions are implicitly imposed in the tangential direction. However, we ensured that the width of the periodic cell $L$ was sufficiently large, and the indentation was sufficiently small such that the contact behaved as isolated. The interfacial loads in the normal and tangential directions are calculated as follows:

$$
\begin{aligned}
& w_{\mathrm{n}}=\int_{0}^{L} p_{\text {int }_{\mathrm{n}}}(x) \mathrm{d} x \\
& w_{\mathrm{t}}=\int_{0}^{L} p_{\text {int }_{\mathrm{t}}}(x) \mathrm{d} x
\end{aligned}
$$

By convention, tensile and compressive loads are indicated by the negative and positive signs, respectively. It is noteworthy that the results will vary slightly if loads are applied instead of displacements. However, for the range of parameters investigated in this study, the difference was insignificant.

\subsection{Traction-separation laws}

As mentioned above, the interaction between the two bodies was modeled using local traction-separation laws. We used these laws to describe the macroscopic behaviors of the interfaces and implicitly incorporated all the nano- and micro-scale features that contributed to them. Although the model can explicitly describe the roughness and nanoscale interactions (such as molecular bonds), we decided not to use such details because they are typically not available from experiments. Traction-separation laws were initially proposed and extensively used in various forms in regard to crack opening [23]. McGarry et al. [19] proposed a formulation, which was also used in Ref. [21], comprising two nonpotential-based coupled laws designed for mixed-mode loading; the results obtained were consistent with the observations, i.e., normal loading enhances friction. The traction-separation laws, represented graphically in Fig. 2, are expressed as follows:

$$
\begin{aligned}
& p_{\text {int }_{\mathrm{n}}}=\frac{\phi_{\mathrm{n}}}{\delta_{\mathrm{n}}} \frac{g_{\mathrm{n}}}{\delta_{\mathrm{n}}} \mathrm{e}^{-g_{\mathrm{n}} / \delta_{\mathrm{n}}} \mathrm{e}^{-\left(g_{\mathrm{t}} / \delta_{\mathrm{t}}\right)^{2}} \\
& p_{\text {int }_{\mathrm{t}}}=\frac{\phi_{\mathrm{t}}}{\delta_{\mathrm{t}}} \frac{g_{\mathrm{t}}}{\delta_{\mathrm{t}}} \mathrm{e}^{-g_{\mathrm{n}} / \delta_{\mathrm{n}}} \mathrm{e}^{-\left(g_{\mathrm{t}} / \delta_{\mathrm{t}}\right)^{2}}
\end{aligned}
$$

where $\phi_{\mathrm{n}}$ and $\phi_{\mathrm{t}}$ are the works of adhesion; $\delta_{\mathrm{n}}$ and $\delta_{\mathrm{t}}$ are the characteristic lengths; $g_{\mathrm{n}}$ and $g_{\mathrm{t}}$ are the gaps between two discretization nodes in different bodies. The maximum values yielded by these functions can be calculated as follows:

$$
\begin{gathered}
\sigma_{\text {max }}=p_{\text {int }_{\mathrm{n}}}\left(g_{\mathrm{n}}=\delta_{\mathrm{n}}, g_{\mathrm{t}}=0\right)=\frac{1}{\mathrm{e}} \frac{\phi_{\mathrm{n}}}{\delta_{\mathrm{n}}} \\
\tau_{\text {max }}=p_{\text {int }_{\mathrm{t}}}\left(g_{\mathrm{n}}=0, g_{\mathrm{t}}=\delta_{\mathrm{t}} / \sqrt{2}\right)=\sqrt{\frac{2}{\mathrm{e}}} \frac{\phi_{\mathrm{t}}}{\delta_{\mathrm{t}}}
\end{gathered}
$$

In the formulation by McGarry et al [19], however, both the normal and tangential pressures approach zero at large separations in either the normal or tangential direction. This condition is realistic when a crack occurs in Mode I. In the context of contact 

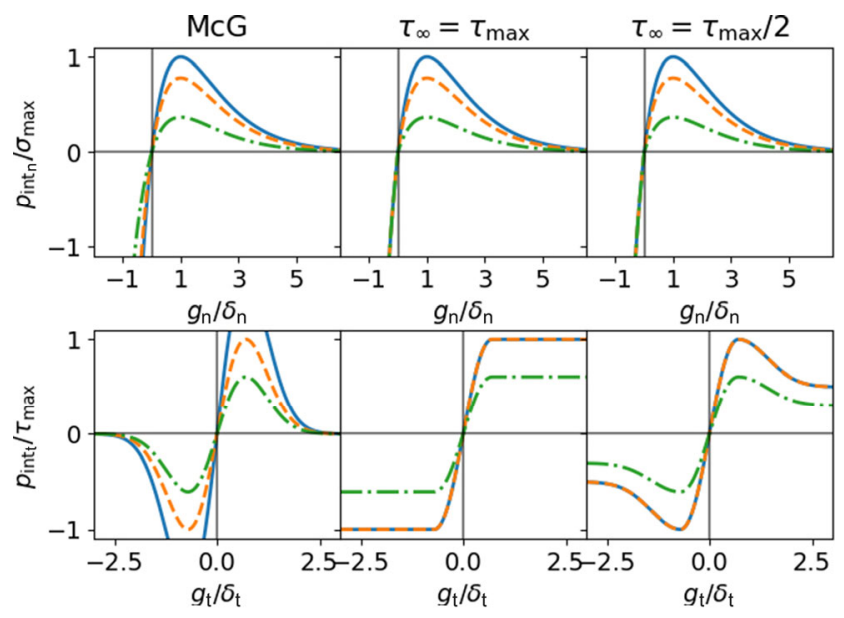

Fig. 2 Representation of the traction-separation laws used in this study. McG denotes the laws proposed by McGarry et al. [19], presented in (3). The other two abbreviations show Eq. (5) for $\tau_{\infty}=\tau_{\max }$ and $\tau_{\infty}=\tau_{\max } / 2$. In the upper row, normal pressure is shown as a function of normal gap for three values of tangential gap: $g_{\mathrm{t}} / \delta_{\mathrm{t}}=0$ (solid blue line), $g_{\mathrm{t}} / \delta_{\mathrm{t}}=0.5$ (dashed orange line), and $g_{\mathrm{t}} / \delta_{\mathrm{t}}=1$ (dash-dotted green line). In the lower row, tangential pressure is shown as a function of tangential gap for three values of normal gap: $g_{\mathrm{n}} / \delta_{\mathrm{n}}=-0.5$ (solid blue line), $g_{\mathrm{n}} / \delta_{\mathrm{n}}=0$ (dashed orange line), and $g_{\mathrm{n}} / \delta_{\mathrm{n}}=0.5$ (dash-dotted green line).

mechanics; however, a frictionless glide will occur if further tangential loading is applied; based on experience, this is an unrealistic situation. Therefore, we modified Eq. (3) to obtain a behavior that is more similar to observations. A traction-separation law that preserves friction and the repulsive normal load at large tangential separations can be written as follows:

$$
\left.\begin{array}{c}
p_{\text {int }_{\mathrm{n}}}=\frac{\phi_{\mathrm{n}}}{\delta_{\mathrm{n}}} \frac{g_{\mathrm{n}}}{\delta_{\mathrm{n}}} \mathrm{e}^{-g_{\mathrm{n}} / \delta_{\mathrm{n}}} H\left(\mathrm{e}^{-\left(g_{\mathrm{t}} / \delta_{\mathrm{t}}\right)^{2}}\right) \\
2 \operatorname{sgn}\left(g_{\mathrm{t}}\right) \frac{\phi_{\mathrm{t}}}{\delta_{\mathrm{t}}} \frac{g_{\mathrm{t}}}{\delta_{\mathrm{t}}} \mathrm{e}^{-\left(g_{\mathrm{t}} / \delta_{\mathrm{t}}\right)^{2}} H\left(\mathrm{e}^{-g_{\mathrm{n}} / \delta_{\mathrm{n}}}\right) \\
\left|g_{\mathrm{t}}\right| \leq \delta_{\mathrm{t}} / \sqrt{2} \\
\operatorname{sgn}\left(g_{\mathrm{t}}\right)\left(\tau_{\infty}+\left(\frac{\phi_{\mathrm{t}} \sqrt{2}}{\delta_{\mathrm{t}} \sqrt{\mathrm{e}}}-\tau_{\infty}\right) \mathrm{e}^{\left(g_{\mathrm{t}} / \delta_{\mathrm{t}}-\operatorname{sgn}\left(g_{\mathrm{t}}\right) / \sqrt{2}\right)^{2}}\right) H\left(\mathrm{e}^{-\delta_{\mathrm{n}} / \delta_{\mathrm{n}}}\right) \\
\left|g_{\mathrm{t}}\right|>\delta_{\mathrm{t}} / \sqrt{2}
\end{array}\right\}
$$

where $\operatorname{sgn}(x)$ outputs the sign of $x ; H(x)=1$ if $g_{\mathrm{n}}<0$ and $H(x)=x$ if $g_{\mathrm{n}}>0$. In this study, we considered only cases where $\tau_{\infty}<\tau_{\max }=\sqrt{1 / 2 \mathrm{e}} \phi_{\mathrm{t}} \delta_{\mathrm{t}}$. Figure 2 shows a comparison between the new curves with those of McGarry et al. [19]. The only difference observed in terms of the normal traction is that the repulsive section of the normal traction remains unchanged when the tangential gap increased, which prevents interpenetration. Meanwhile, the difference observed in terms of the tangential traction is that the load levels at a finite value $\tau_{\infty}$ instead of at zero, unlike the formulation proposed by McGarry et al. [19]. It is noteworthy that selecting a non-zero value for $\tau_{\infty}$ results in an unbounded energy required to displace the body tangentially to infinity against the friction force. This should be interpreted as follows: The traction-separation curve is segregated in two regions. The first region, where $\left|g_{t}\right| \leq \delta_{t}$, represents a reversible stretching of the bonds formed between two surfaces, with a finite energy $\phi_{\mathrm{t}}$ associated to it; the second region, where $\left|g_{t}\right| \leq \delta_{t} / \sqrt{2}$ represents irreversible frictional sliding.

\subsection{Dimensionless formulations}

To reduce the number of parameters, we set the problem in dimensionless form by adopting the following scaled parameters:

$$
\begin{aligned}
& X=\frac{x}{x_{\mathrm{r}}}, P_{\mathrm{n}}=\frac{p_{\mathrm{n}}}{p_{\mathrm{n}_{\mathrm{r}}}}, P_{\mathrm{t}}=\frac{p_{\mathrm{t}}}{p_{\mathrm{t}_{\mathrm{r}}}}, U_{\mathrm{n}}=\frac{u_{\mathrm{n}}}{u_{\mathrm{n}_{\mathrm{r}}}}, U_{t}=\frac{u_{t}}{u_{\mathrm{t}_{\mathrm{r}}}}, \\
& W_{\mathrm{n}}=\frac{w_{\mathrm{n}}}{w_{\mathrm{n}_{\mathrm{r}}}}, W_{\mathrm{t}}=\frac{w_{\mathrm{t}}}{w_{\mathrm{t}_{\mathrm{r}}}}
\end{aligned}
$$

where the variables with subscript $r$ are reference parameters, defined by

$$
\begin{aligned}
& x_{\mathrm{r}}=\beta=\left(\frac{R^{2} \phi_{\mathrm{n}}}{E^{*}}\right)^{1 / 3}, u_{\mathrm{n}_{\mathrm{r}}}=\frac{\beta^{2}}{R}, p_{\mathrm{n}_{\mathrm{r}}}=\frac{\phi_{\mathrm{n}}}{\delta_{\mathrm{n}}}, w_{\mathrm{n}_{\mathrm{r}}}=\left(E^{*} R \phi_{\mathrm{n}}^{2}\right)^{1 / 3} \\
& p_{\mathrm{t}_{\mathrm{r}}}=\frac{\phi_{\mathrm{t}}}{\delta_{\mathrm{t}}}, u_{\mathrm{t}_{\mathrm{r}}}=\sqrt{\phi_{\mathrm{t}} / \phi_{\mathrm{n}}} \frac{\beta^{2}}{R}, w_{\mathrm{t}_{\mathrm{r}}}=\sqrt{\phi_{\mathrm{t}} / \phi_{\mathrm{n}}}\left(E^{*} R \phi_{\mathrm{n}}^{2}\right)^{1 / 3}
\end{aligned}
$$

where $E^{*}=E /\left(1-v^{2}\right)$. It is noteworthy that the scaling used for the parameters in the normal direction is based on that used in Ref. [24]. Using this scaling, the relevant equations for the problem are expressed as follows:

$$
p_{\mathrm{el}_{\mathrm{n}}}=\frac{1}{2 \lambda_{\mathrm{n}}}\left(U_{\mathrm{n}}^{\mathrm{s}} \tilde{G}_{44}+U_{\mathrm{app}_{\mathrm{n}}} \tilde{G}_{24}\right)
$$




$$
\begin{gathered}
p_{\mathrm{el}_{\mathrm{t}}}=\frac{1}{2 \lambda_{\mathrm{t}}}\left(U_{\mathrm{t}}^{\mathrm{s}} \tilde{G}_{33}+U_{\mathrm{app}_{\mathrm{t}}} \tilde{G}_{13}\right) \\
G_{0}=\frac{X^{2}}{2}+\frac{\bar{G}_{0}}{\lambda_{\mathrm{n}}} \\
G_{\mathrm{n}}=G_{0}+U_{\mathrm{n}}^{\mathrm{s}} \\
G_{\mathrm{t}}=U_{\mathrm{t}}^{\mathrm{s}} \\
p_{\text {int }_{\mathrm{n}}}=p_{\text {int }_{\mathrm{n}}}\left(G_{\mathrm{n}}, G_{\mathrm{t}}, \lambda_{\mathrm{n}}, \lambda_{\mathrm{t}}\right) \\
p_{\text {int }_{\mathrm{t}}}=p_{\text {int }_{\mathrm{t}}}\left(G_{\mathrm{n}}, G_{\mathrm{t}}, \lambda_{\mathrm{n}}, \lambda_{\mathrm{t}}\right) \\
W_{\mathrm{n}}=\lambda_{\mathrm{n}} \int_{0}^{\frac{L}{x_{\mathrm{r}}}} p_{\text {int }_{\mathrm{n}}}(X) \mathrm{d} X \\
W_{\mathrm{t}}=\lambda_{\mathrm{t}} \int_{0}^{\frac{L}{x_{\mathrm{r}}}} p_{\text {int }_{\mathrm{t}}}(X) \mathrm{d} X
\end{gathered}
$$

where $G_{0}$ is the initial (normal) gap, and $\tilde{G}_{i j}=G_{i j} x_{\mathrm{r}} / E^{*}$ is the dimensionless Green's function. In Eqs. (9a) and (9b), only terms that do not vanish, when the height of the bodies is extremely large, are included. These terms vanish only because we assume that the elastic body is incompressible. Otherwise, they will be present and a more complex problem will be encountered, with an additional parameter describing the coupling strength between normal pressures and tangential deformations, and vice versa.

Owing to this normalization, the response of the contacting body to a specified loading is determined by only two parameters, $\lambda_{\mathrm{n}}$ and $\lambda_{\mathrm{t}}$, defined as follows:

$\lambda_{\mathrm{n}}=\frac{u_{\mathrm{n}_{\mathrm{r}}}}{\delta_{\mathrm{n}}}=\frac{\phi_{\mathrm{n}}}{\delta_{\mathrm{n}}}\left(\frac{R}{\left(E^{*}\right)^{2} \phi_{\mathrm{n}}}\right)^{\frac{1}{3}}, \lambda_{\mathrm{t}}=\frac{u_{\mathrm{t}_{\mathrm{r}}}}{\delta_{\mathrm{t}}}=\frac{\sqrt{\phi_{\mathrm{n}} \phi_{\mathrm{t}}}}{\delta_{\mathrm{t}}}\left(\frac{R}{\left(E^{*}\right)^{2} \phi_{\mathrm{n}}}\right)^{\frac{1}{3}}$

where $\lambda_{\mathrm{n}}$ is the well-known Maugis parameter (also known as Tabor's parameter) [24]. When only normal contact is considered, the limit $\lambda_{\mathrm{n}} \rightarrow \infty$ signifies shortrange adhesion, as compared with the normal elastic deformations, and corresponds to the Johnson, Kendall, and Roberts (JKR) solution. Here, $\lambda_{t}$ is defined equivalently for the tangential direction and should therefore be regarded similarly, i.e., large values of $\lambda_{\mathrm{t}}$ correspond to short-range friction. More precisely, large values of $\lambda_{\mathrm{t}}$ indicate that the elastic deformation induced by the loading in the body are insignificant as compared with the length scale of the tangential separation allowed by the interface, $\delta_{\mathrm{t}}$. When $\phi_{\mathrm{t}}=\phi_{\mathrm{n}}$ and $\delta_{\mathrm{t}}=\delta_{\mathrm{n}}$, it was found that $\lambda_{\mathrm{n}}=\lambda_{\mathrm{t}}$, as expected. Additionally, it is noteworthy that $\phi_{\mathrm{n}}$ appears, perhaps unexpectedly, in the definition of $\lambda_{\mathrm{t}}$ : An increase in $\phi_{\mathrm{n}}$ results in an increase in the contact area; the contact enables a larger tangential load to be sustained and hence more significant elastic deformations prior to sliding. We comprehensively investigated the effect of $\lambda_{\mathrm{t}}$ on the results of this study.

It is noteworthy that the results depend on $H / x_{\mathrm{r}}$ and $L / x_{\mathrm{r}}$. However, the value selected for $H$ ( $H \approx 2 \times 10^{3} x_{\mathrm{r}}$ ) was sufficiently large to guarantee that a further increase in $H$ would only result in a decrease in the total force resulting from an applied displacement. This will not affect the results, which are presented in terms of forces and applied displacements. Similarly, $L \approx 100 x_{\mathrm{r}}$ was chosen, which is sufficiently large to ensure that the contact behaves as isolated. Therefore, the dependences of the results on $H / x_{\mathrm{r}}$ and $L / x_{\mathrm{r}}$ were not considered.

\subsection{Definition of contact area}

The definition of the contact area becomes nontrivial when adhesion is present. Without adhesion, the contact area can be defined as the area in which the contact pressure is greater than zero. When adhesion is present, however, the pressure is negative when there is a positive gap between the surfaces, but these are close enough to feel each other's influence. To facilitate a meaningful comparison with analytical theories and experimental results, a fraction of the surface under adhesion must be considered when computing the contact area.

Analytical theories, such as JKR, often include all adhesive components in the definition of the contact area. In this theory, a singularity occurs at the edge of the contact, rendering the total contact area well defined. Moreover, when comparing the results with experimental values, one should be aware of the parameters detected by the measuring technique used. For example, in the experimental setup used by Sahli et al. [5], the contact area was measured owing to the different reflective properties of light when it is in 
contact or otherwise with the interface. It is clear that separations smaller than the wavelength of the light used $(\sim 500 \mathrm{~nm})$ will be blended in with the contact. Therefore, we also included on the definition of the contact area parts of the surfaces that are under attractive interaction when the separation is small.

In this regard, a separation threshold must be defined so that points are in considered to be in contact if their separation is below the threshold. This threshold should be in the order of $\delta_{\mathrm{n}}$, as this is the length scale at which adhesive forces act. As shown in Fig. 3, the threshold value affected the calculated contact area, although convergence was observed for sufficiently large values. In this study, we selected a threshold equal to $\mathrm{e} \delta_{\mathrm{n}}$, which yielded results that were similar to the JKR solution. As shown in the inset of Fig. 3(a), the work of adhesion in the model corresponds to the work caused by a constant pressure $\sigma_{\max }$ from 0 to the selected threshold.

\section{Selection of Maugis parameter $\lambda_{\mathrm{n}}$}

The model presented herein allows us to vary $\lambda_{\mathrm{n}}$ to consider the entire range between the JKR and DMT limits. However, to ensure a low number of cases, we fixed $\lambda_{\mathrm{n}}$. Indentation was performed to select a suitable value. Because the bodies were assumed to be incompressible, normal loading caused no displacement in the tangential direction, and the results were therefore independent of $\lambda_{t}$. In most experiments performed with PDMS, the increase in contact area during normal loading was similar to the JKR solution [2]. Therefore, we selected an appropriate value of $\lambda_{\mathrm{n}}$ to reproduce the JKR solution, except for the singularity of the tractions at the edge of the contact. Figure 4(a) shows the evolution of the contact area and the pressure distribution obtained for $\lambda_{\mathrm{n}}=$ 3,10 , and 20. Focusing on the area, the difference was insignificant for the three larger values of $\lambda_{\mathrm{n}}$ (less
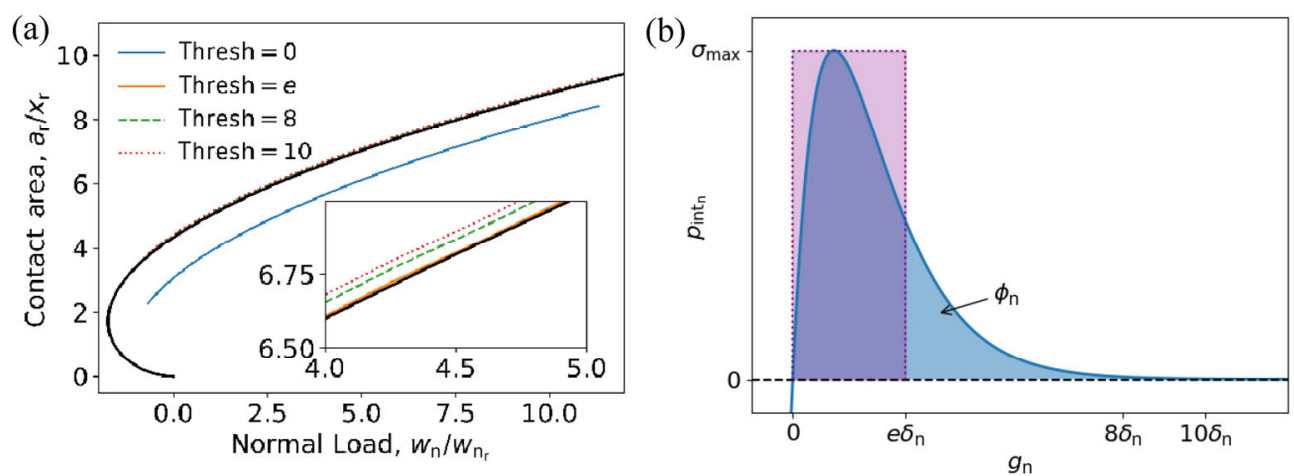

Fig. 3 (a) Contact area for various thresholds. The value tresh $=0$ corresponds to consideration of only the repulsive section of the contact area. The values tresh $=8 \delta_{\mathrm{n}}$ and tresh $=10 \delta_{\mathrm{n}}$ correspond to when $p_{\text {int }_{\mathrm{n}}}$ decreased to $1 \%$ and $0.1 \%$ of their maximum value, respectively. The interpretation of the value tresh $=\mathrm{e} \delta_{\mathrm{n}}$ is shown in (b), where the two shaded areas are both equal to the work of adhesion $\phi_{\mathrm{n}}$.
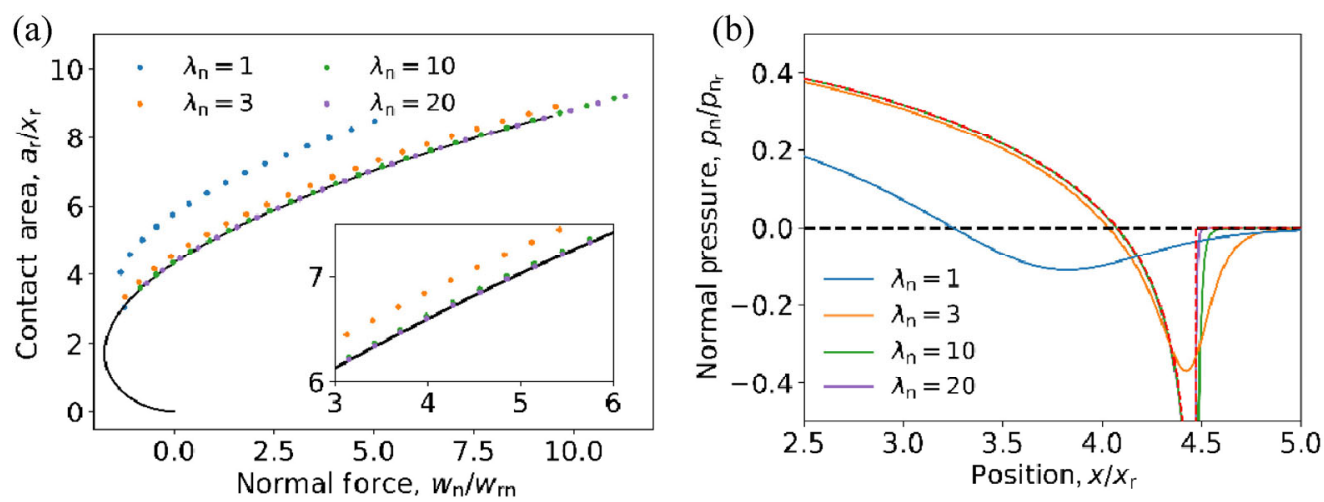

Fig. 4 (a) Contact area vs. normal load for three values of $\lambda_{n}$. Black line corresponds to the JKR solution. The inset shows the zoomed-in section of curve and (b) pressure distribution for the same cases, at a load of $w_{\mathrm{n}} / w_{\mathrm{n}_{\mathrm{r}}}=10.4$. Dashed red line corresponds to the JKR solution. 
than $5 \%$ ), whereas it increased as $\lambda_{\mathrm{n}}$ increased. This is to be expected, as Johnson and Greenwood [24] reported that $\lambda_{\mathrm{n}}>3$ was sufficient to obtain a converged result for the contact area.

The pressure distribution together with the JKR solution is shown in Fig. 4(b). The numerical curves were similar to the analytical curves except at the edge of the contact, where the analytical solution is singular, and the numerical solutions exhibited a pressure peak that was broader and lower for smaller values of $\lambda_{\mathrm{n}}$. To accurately describe this peak, a $\lambda_{\mathrm{n}}$ value larger than that required for computing the contact area is required. Therefore, we selected $\lambda_{\mathrm{n}}=10$. It is noteworthy that we did not select a higher value of $\lambda_{\mathrm{n}}$ to avoid a finer discretization. In this study, we used $2^{13}$ points to discretize the surface.

\section{Effect of tangential Maugis-like parameter $\lambda_{\mathrm{t}}$}

Based on a fixed value of $\lambda_{\mathrm{n}}=10$, we investigated the effect of three different tangential Maugis parameters, i.e., $\lambda_{\mathrm{t}}=10, \lambda_{\mathrm{t}}=1$, and $\lambda_{\mathrm{t}}=0.1$. It is noteworthy that $\lambda_{\mathrm{t}}<\lambda_{\mathrm{n}}$ indicates that $\phi_{\mathrm{t}}<\phi_{\mathrm{n}}$ and/or $\delta_{\mathrm{t}}>\delta_{\mathrm{n}}$. We first considered the area evolution with increasing applied tangential displacement $u_{\text {app }_{\mathrm{t}}}$, as presented in Fig. 5(a). Two values of the normal displacement were applied, i.e., $u_{\text {app }_{\mathrm{t}}} / u_{\mathrm{n}_{\mathrm{r}}}=40$ and $u_{\text {app }_{\mathrm{t}}} / u_{\mathrm{n}_{\mathrm{r}}}=2$, which resulted in normal forces of $w_{n} / w_{n_{r}}=25$ and $w_{n} / w_{n_{r}}=0.8$, respectively. For both normal loads, when $\lambda_{t}=0.1$, the contact area decreased rapidly after tangential loading was applied. Subsequently, gross sliding commenced unstably (the displacement at which occurred is indicated in Fig. 5(a) with a marker), as adhesion vanished abruptly. By contrast, when $\lambda_{\mathrm{t}}=10$, the contact area decreased slowly until the onset of gross sliding. As indicated by the marker, the minimum contact area, which indicates that adhesion has completely vanished, was attained prior to the onset of gross sliding; hence, the contact area did not decrease abruptly. However, it is noteworthy that the transition toward gross sliding remained unstable because $\tau_{\infty}<\tau_{\max }$. The case with $\lambda_{\mathrm{t}}=1$ was similar to the case with $\lambda_{\mathrm{t}}=10$, although the presence of an abrupt decrease in the contact area at the transition toward gross sliding depended on the magnitude of the normal load. When the load was low, an abrupt decrease was observed, although it was much smaller than that of the case with $\lambda_{\mathrm{t}}=0.1$. However, when the load was higher, such an abrupt decrease was not observed. This dependence on the load is due to the contact area; a larger area can sustain larger tangential stresses prior to gross sliding. This larger tangential load results in a more significant deformation at the edge of the contact, which increases the tangential gap locally and reduces adhesion. It is noteworthy that, in the experimental studies by Mergel et al. [2] and Sahli et al. [5], it was observed that the contact area decreased after the onset of gross sliding, although it was less abrupt compared with the observations of the current study. For all values of $\lambda_{\mathrm{t}}$, after gross sliding began, the contact area remained constant, which is consistent with the results presented in Ref. [3]. However, in the study presented in Ref. [2], where the setup used was the same as that reported in Ref. [3] but with a displacement applied at a higher rate, a slight decrease in contact area was observed. This is attributed to the viscoelastic effects, which were not captured by the current model.

Furthermore, it is unclear whether adhesion vanishes completely or whether some remnant adhesive pressure prevails at large tangential separations. To capture the remnant adhesive pressure, one can modify the traction-separation law by prescribing the normal traction to yield a non-zero value at large tangential displacements. With this modification, the results will not change qualitatively, but the contact area will stabilize at a slightly higher value.

Figure 5(b) shows a plot of the total tangential force against displacement. As the applied displacement increased, the tangential force increased almost linearly, and the response was primarily governed by the elasticity of the body. Eventually, gross sliding occurred, and the tangential force decreased abruptly. This abrupt decrease is attributed to the following two reasons: (i) $\tau_{\infty}<\tau_{\max }$, which implies that the tangential stress that can be withstood by the interface during slip is smaller than that at its onset; (ii) the contact area decreased abruptly. The tangential force at which sliding occurs depends on both $w_{\mathrm{n}}$ and $\lambda_{\mathrm{t}}$. It is noteworthy that increasing $w_{\mathrm{n}}$ results in a larger contact area. 
(a)

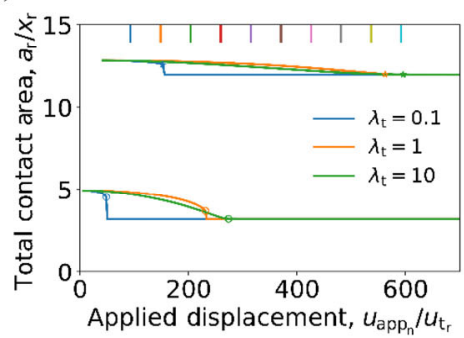

(d)

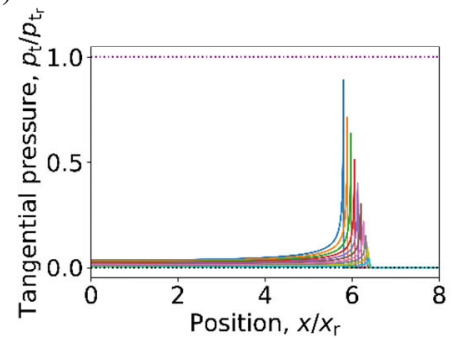

(b)

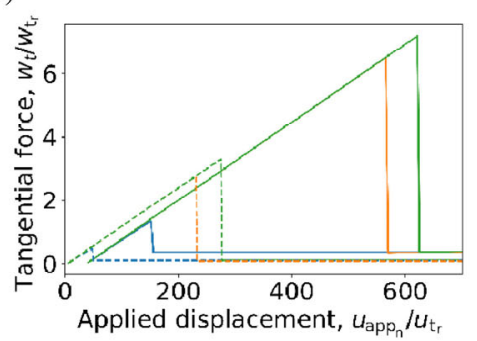

(e)

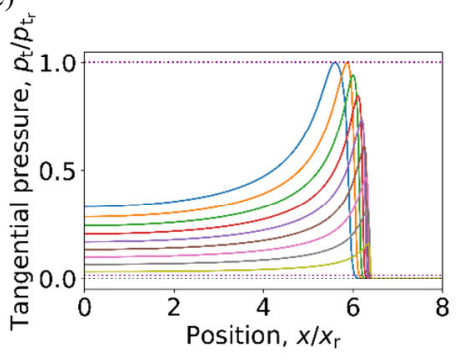

(c)

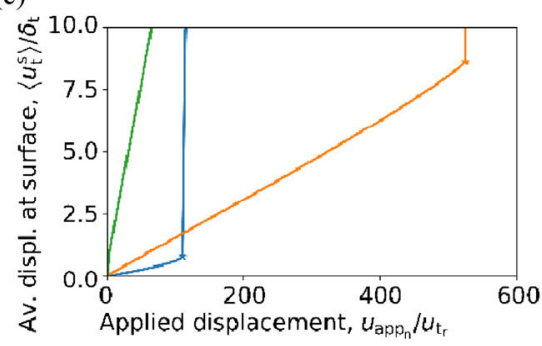

(f)

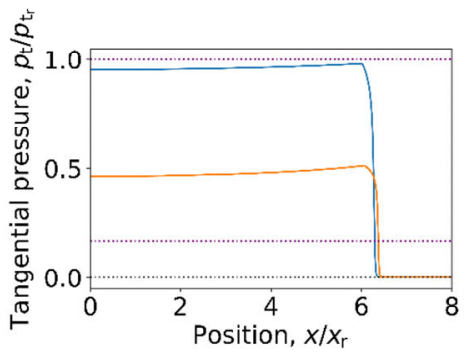

Fig. 5 (a) Contact area vs. tangential displacement for three values of $\lambda_{t}$. Two values of applied normal displacements are shown: $u_{\text {app }_{\mathrm{n}}} / u_{\mathrm{n}_{\mathrm{r}}}=40\left(w_{\mathrm{n}} / w_{\mathrm{n}_{\mathrm{r}}}=25\right)$ and $u_{\text {app }_{\mathrm{n}}} / u_{\mathrm{n}_{\mathrm{r}}}=2\left(w_{\mathrm{n}} / w_{\mathrm{n}_{\mathrm{r}}}=0.8\right)$. Markers indicate the onset of gross sliding (stars for $w_{\mathrm{n}} / w_{\mathrm{n}_{\mathrm{r}}}=25$, and circles for $w_{\mathrm{n}} / w_{\mathrm{n}_{\mathrm{r}}}=0.8$ ). (b) Tangential force vs. tangential displacement for the same cases as in (a). Solid and dashed lines correspond to $u_{\text {app }_{\mathrm{n}}} / u_{\mathrm{n}_{\mathrm{r}}}=40\left(w_{\mathrm{n}} / w_{\mathrm{n}_{\mathrm{r}}}=25\right)$ and $u_{\text {app }_{\mathrm{n}}} / u_{\mathrm{n}_{\mathrm{r}}}=2\left(w_{\mathrm{n}} / w_{\mathrm{n}_{\mathrm{r}}}=0.8\right)$, respectively. (c) The ratio $u_{\mathrm{t}}^{\mathrm{s}} / \delta_{\mathrm{t}}$ for the force $w_{\mathrm{n}} / w_{\mathrm{n}_{\mathrm{r}}}=25$, where $u_{\mathrm{t}}^{\mathrm{s}}$ indicates the average displacement at the surface. (d-f) Tangential pressure profile for the indicated tractionseparation laws and the three values of $\lambda_{\mathrm{t}}$ used in $(\mathrm{a}-\mathrm{c})$. In all cases, $w_{\mathrm{n}} / w_{\mathrm{n}_{\mathrm{r}}}=25$, and the load corresponds to the small vertical lines in (a). Dotted purple lines indicate $\tau_{\max }$ and $\tau_{\infty}$.

To clarify the effect of $\lambda_{\mathrm{t}}$ on the manner in which the contact area is reduced, it is instructive to consider the compliance of the system. The displacement at the contact is governed by the compliance of the body and that of the interface. If they are of the same order, as is the case with small values of $\lambda_{t}$, then the compliance of the interface can accommodate some interfacial displacements before sliding. This situation resembles ductile Mode II failure. By contrast, if the compliance of the interface is much smaller, any local point will shift rapidly from zero displacements to slip. In this case, the onset of slip resembles brittle Mode II failure. To visualize the relative compliance of the contacts based on different values of $\lambda_{t}$, we plotted the ratio $u_{\mathrm{t}}^{\mathrm{s}} / \delta_{\mathrm{t}}$ (Fig. 5(c)), where $u_{\mathrm{t}}^{\mathrm{s}}$ is the average of the deformation at the surface, including both contact and non-contact regions. Hence, the ratio provides the relative displacement of the body with respect to the displacement of the interface. It is noteworthy that $\delta_{\mathrm{t}}$ is the length scale of the traction-separation law and therefore provides the upper limit to the deformations that the contact can accommodate prior to an irreversible slip. Hence, a high value of this ratio, e.g., $\lambda_{t}=10$, indicates that the contact interface is extremely stiff as compared with the body. For smaller values of $\lambda_{t}$, particularly $\lambda_{t}=0.1$, the compliance of the interface becomes much more significant. In this case, the change in the slope corresponds to gross sliding.

Images of the pressure profile are shown in Figs. 5(d)-5(f) for the three abovementioned values of $\lambda_{\mathrm{t}}$. The colors of the curves correspond to the values of the applied tangential displacement, as indicated in Fig. 5(a). The pressure values were normalized by $p_{\mathrm{t}_{\mathrm{r}}}=\phi_{\mathrm{t}} / \delta_{\mathrm{t}}=\tau_{\max }$, which increases with $\lambda_{\mathrm{t}}$. At high values of $\lambda_{t}$ (Fig. 5(d)), the low compliance of the interface resulted in high stresses, which formed a spike near the edge of the contact. As soon as the spike reached the value of $\tau_{\max }$, the abrupt decrease in the tangential pressure toward $\tau_{\infty}$ triggered an early unstable transition toward gross sliding. For $\lambda_{\mathrm{t}}=1$, the additional compliance of the interface resulted in a shallower, rounder spike. Because the stresses at the edge of the contact were smaller, the tangential displacements and decay in the contact area occurred at a slower pace. Finally, when $\lambda_{\mathrm{t}}=0.1$, the compliance of the interface was extremely large, resulting in a 
constant pressure profile. Finally, it is noteworthy that $\tau_{\infty}$ did not significantly affect the incipient tangential loading prior to gross sliding.

To better visualize the comparison with the experimental results presented by Refs. [2, 5], the simulation results shown in Fig. 5(a) are presented in Fig. 6 by correlating the contact area with the tangential force instead of with the applied displacement. It is noteworthy that more values of $\lambda_{\mathrm{t}}$ were added, and a non-monotonic trend was observed, similar to Fig. 5. For $\lambda_{\mathrm{t}}>1$, an increase in $\lambda_{\mathrm{t}}$ resulted in a more rapid decrease in the contact area. As shown in Fig. 5, an increase in $\lambda_{\mathrm{t}}$ resulted in a higher but narrower pressure spike at the contact edge and a faster peeling toward the center, and hence a faster decay of the contact area. Eventually, convergence was observed when $\lambda_{\mathrm{t}} \approx 10$. It is noteworthy that the convergence of the normal component occurred at $\lambda_{\mathrm{n}} \approx 10$ as well. However, when $\lambda_{\mathrm{t}}<1$, the contact area decreased with $\lambda_{\mathrm{t}}$. In this case, the pressure profile was similar to that shown in Fig. 5(f), i.e., the tangential pressure profile was relatively constant. In this case, the load that can be sustained by the contact was limited by $\tau_{\max } a_{\mathrm{r}}$. Because $\tau_{\max }$ decreased with $\lambda_{\mathrm{t}}$, an early transition toward gross sliding was observed.

The superimposed dotted gray lines indicate the best fit to the power law. In all cases, the exponent was approximately 2 (it was between 1.8 and 2.2 in all the cases considered). Such a power-law behavior, as well as the measured exponent, is consistent with the experimental observations presented in Refs. [2, 5]. However, our results are consistent with the power law only at low tangential loads. For high tangential loads, this law is only suitable for some values of the normal load and $\lambda_{\mathrm{t}}$. When $w_{\mathrm{n}} / w_{\mathrm{n}_{\mathrm{r}}}=25$, the best agreement was observed when $\lambda_{\mathrm{t}}=1$. For lower values, the decay was faster than that of the power law. For higher values, the fit was excellent initially; however, the contact area formed a tail with a slower decay. By contrast, when $w_{\mathrm{n}} / w_{\mathrm{n}_{\mathrm{r}}}=0.8$, the decay was too fast to conform to the power law for $\lambda_{t}<10$. However, at higher values, the agreement was the best.

Finally, we focused on the effect of normal loading on the relationship between the contact area and tangential force. Figure 7 shows the evolution of the contact area as the tangential force increased until the
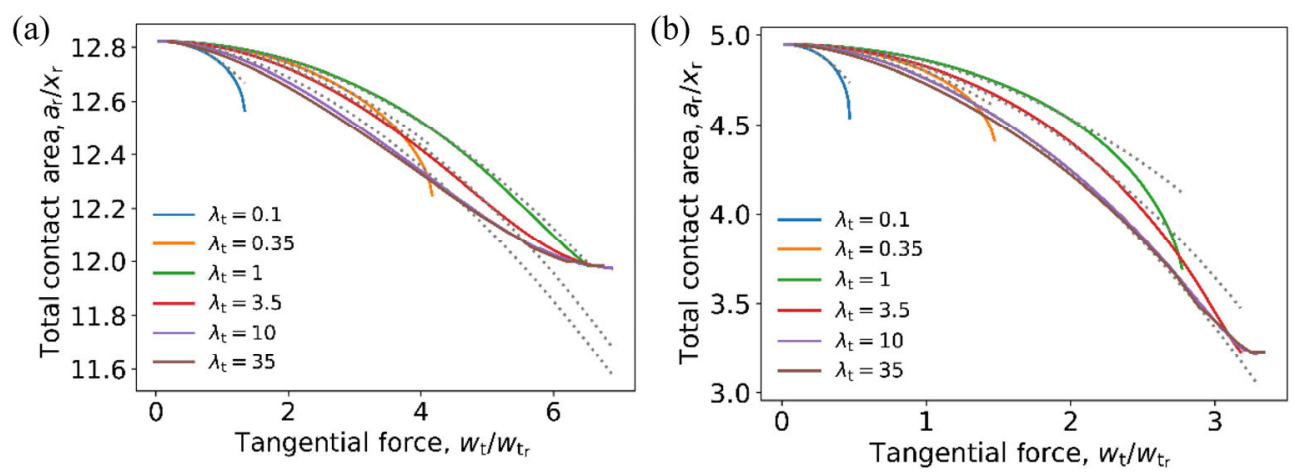

Fig. 6 Decay in contact area for indicated load and various $\lambda_{t}$ values. Dotted gray lines show the best fit with power law, with exponent ranging from 1.8 to 2.2 for all cases.

(a)

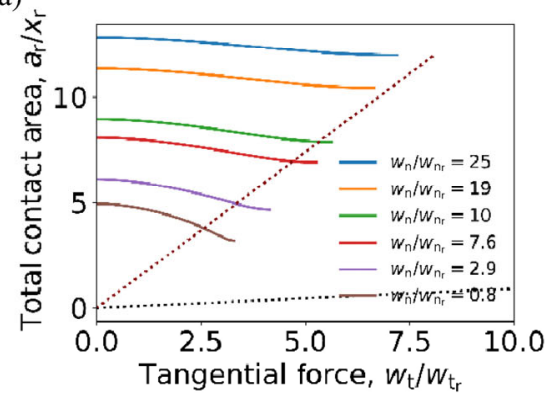

(b)

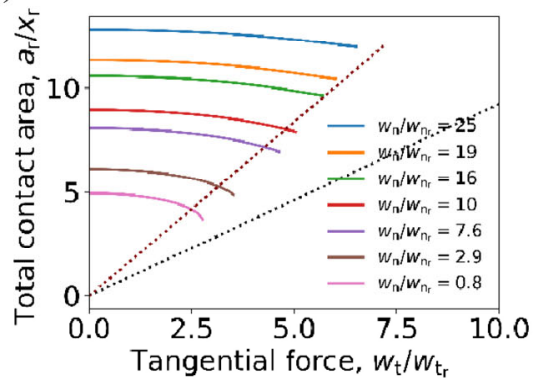

(c)

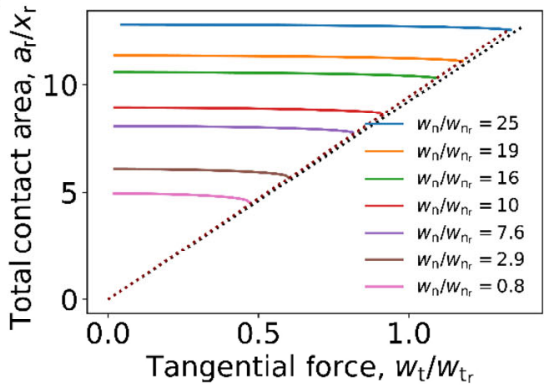

Fig. 7 Decay in contact area with increasing tangential force for $\lambda_{\mathrm{t}}=0.1$ and the indicated traction-separation laws. Dotted black line indicates the line $w_{\mathrm{t}}=\tau_{\max } a_{\mathrm{r}}$, whereas red ones indicate the same relation but with $\tau_{\text {fit }}$ fitted from the data. 
onset of gross sliding. When $\lambda_{t}=0.1$ with different normal forces was considered, all the points representing the start of gross sliding can be fitted with a straight line passing through the origin. Therefore, we can write $w_{\mathrm{t}}^{\mathrm{o}}=\tau_{\mathrm{fit}} a_{\mathrm{r}}^{\mathrm{o}}$, where the superscript o indicates the point at which the onset occurs, and $\tau_{\text {fit }} \approx \tau_{\text {max }}$. This is because the tangential pressure is approximately equal to $\tau_{\max }$ in the contact area immediately prior to the onset of gross sliding. As $\lambda_{t}$ increases, a line can still be fitted, albeit not the fit is not as good. It is noteworthy that $\tau_{\text {fit }}$ differs significantly from $\tau_{\max }$. This is because unlike in the case with $\lambda_{\mathrm{t}}=0.1, \tau_{\max }$ is only attained at the edge of the contact, and the tangential pressure is much lower in other locations.

The linear relation obtained between the contact area, and tangential force is consistent with the experimental observations reported in Refs. [2, 5-7] as well as with the interpretation of Amonton's law. In fact, several authors (e.g., Refs. [18, 25]) expressed the friction force as follows:

$$
w_{\mathrm{t}}=\tau_{\mathrm{fit}} a_{\mathrm{r}}+\alpha w_{\mathrm{n}}
$$

where the constant term is attributed to adhesion, and the term linear with $w_{\mathrm{n}}$ arises from the presence of roughness. In our case, the ball was smooth, and only the first term was present. Experimentally, it was observed that $\tau_{\text {fit }}$ should increase with the work of adhesion [25]. In our case, assuming that $\phi_{\mathrm{t}}$ and $\phi_{\mathrm{n}}$ are of the same order of magnitude, we observe that $\tau_{\text {fit }}$ should scale as $\phi_{\mathrm{n}}^{1 / 3}$. Furthermore, $\tau_{\text {fit }}$ increases $\lambda_{\mathrm{t}}$, which increases with $\phi_{\mathrm{n}}$. Hence, our results are qualitatively consistent with those reported in Ref. [25]. However, it is noteworthy that an exponential law was used in Ref. [25] to fit the experimental results correlating $\tau_{\text {fit }}$ and $w_{\mathrm{n}}$.

To summarize, we can conclude that the case with $\lambda_{\mathrm{t}}=0.1$ showed the best agreement with experimental observations from Refs. $[2,5,6]$ when focusing only on the onset of gross sliding. However, the contact area decay was too rapid. Hence, the cases with $\lambda_{t}=1$ and $\lambda_{\mathrm{t}}=10$ provided a better overall agreement.

\section{Discussion}

The model presented herein can qualitatively replicate experimentally observed results from Refs. $[2,5,6]$. Unfortunately, it presents several limitations that disallow a quantitative comparison with the experiments. Because our model is two-dimensional, it might overlook some inherent three-dimensional features. For instance, it has been shown experimentally that a decreasing contact area does not maintain the radial symmetry of the initial contact [6]. In addition, interface behaviors such as reattachment, which was considered in Ref. [20], are not captured by the model. Therefore, the stick-slip behavior of the system cannot be captured. Similarly, the rate dependencies of the properties at the interface [26] were not considered. In addition, because our model is limited to quasi-static elastic deformations, it cannot capture some phenomena, such as viscoelastic effects [2] or dynamic Schallamach waves [4, 27-29]. Finally, it is clear that our model specifies, by construction, that the contact area should be reduced by increasing the tangential load. While this is typically observed experimentally, we point out that, in some studies (see Krick et al. [30] and Menga et al. [31]), an increase was observed. This is attributed to viscoelastic effects, which were not considered in this study.

It is instructive to compare our model with those that addressed the same problem using the concepts of LEFM. These models have been used successfully to replicate experimental observations and yielded compact, nearly closed-form solutions that are convenient to use. However, our method does not prespecify the geometry of the contacting bodies and can hence manage problems other than cylinder-on-flat or ball-on-flat contacts. It is noteworthy that, similar to our model, all these models have fitting parameters that are needed for them to replicate the experimental observations.

The first model based on LEFM was Savkoor's model [10], which assumes that a negligible slip occurs when the contact area decreases. More precisely, it is assumed that the tangential stress field increases in a square root manner toward the contact edge. The singularity at the edge was avoided owing to a small process zone surrounding the contact edge. This implies that any possible (small) slip must be confined within this small process zone. This model can be considered as the tangential counterpart to JKR theory. To improve this model, which was found to predict a too rapid decay 
of the contact area, two approaches were adopted. In the first approach [4, 12, 13], the assumption of negligible slip is maintained, and based on the idea of Hutchinson [32], a mixed-mode function is introduced to capture the decrease in the work of adhesion during tangential loading more accurately. This resulted in a slower decrease in the contact area. The second approach, pioneered by Johnson [11] and further extended by McMeeking et al. [14], and Ciavarella and Papangelo [15], allows slip and assumes that a portion of this slip is reversible. This reversible slip accumulates energy at the interface and hence reduces the work of adhesion. Consequently, the contact area is reduced. Johnson [11] argued that no reversible slip should be expected after a certain amount of slip $s_{0}$, because the mechanism to accumulate energy saturates. However, the effects of this limit have not been investigated comprehensively. Despite the differences in these two approaches, both yielded equivalent results under the appropriate mixed-mode function [15].

Hence, our model can be regarded having characteristics of the two abovementioned approaches. In fact, the coupled traction-separation laws in our model have similarities with the mixed-mode function proposed by Hutchinson [32]; additionally, they allow for a slip that can be separated into reversible and irreversible components, which is consistent with the approach pioneered by Johnson. At low values of $\lambda_{\mathrm{t}}$, our model predicted a negligible slip. In this case, the assumptions made in Hutchinson's approach application. At higher values of $\lambda_{t}$, slip occurs instead, and the results are more similar to those of Johnson's approach. However, our model and Johnson's approach differed significantly: The slip in our model is fully reversible for tangential gaps smaller than $\delta_{\mathrm{t}} / \sqrt{2}$ and fully irreversible otherwise, whereas in Johnson's approach, both reversible and irreversible slip occurs at a fixed ratio [11, 14, 15]. In reality, one can expect dissipation to begin as soon as slip occurs, with most of the slip being reversible initially and the irreversible component increasing rapidly until it dominates at large distances.

As discussed earlier, the experimental results were successfully replicated qualitatively within a certain range of $\lambda_{\mathrm{t}}$ and for high values of $\lambda_{\mathrm{n}}$. The definition of these parameters includes length scales $\delta_{\mathrm{n}}$ and $\delta_{\mathrm{t}}$, which are difficult to measure in macroscopic experiments. One may expect them to be associated with the dominant features at the interface. These lengths can be estimated from the known quantities and the values of $\lambda_{\mathrm{n}}$ and $\lambda_{\mathrm{t}}$. Based on Eq. (10), $\delta_{\mathrm{n}}$ and $\delta_{\mathrm{t}}$ can be expressed as

$$
\delta_{\mathrm{n}}=\frac{\phi_{\mathrm{n}}}{\lambda_{\mathrm{n}}}\left(\frac{R}{\left(E^{*}\right)^{2} \phi_{\mathrm{n}}}\right)^{1 / 3}, \delta_{\mathrm{t}}=\frac{\sqrt{\phi_{\mathrm{t}} \phi_{\mathrm{n}}}}{\lambda_{\mathrm{t}}}\left(\frac{R}{\left(E^{*}\right)^{2} \phi_{\mathrm{n}}}\right)^{1 / 3}
$$

If we set $\phi_{\mathrm{n}}=30 \mathrm{mN} / \mathrm{m}, \phi_{\mathrm{t}}=30 \mathrm{mN} / \mathrm{m}, E^{*}=2 \mathrm{MPa}$, and $R=10 \mathrm{~mm}$, which are similar to those in Refs. [2, 5], as well as assuming $\lambda_{\mathrm{n}} \gtrsim 3$ and $\lambda_{\mathrm{t}}$ ranging from 1 to 3.6 (according to Figs. $4(\mathrm{a})$ and 6 ), then $\delta_{\mathrm{n}} \lesssim 0.4 \mu \mathrm{m}$ and $\delta_{\mathrm{t}} \approx 0.6 \mu \mathrm{m}$ are obtained. These lengths can be correlated with the surface roughness, which exists inevitably on macroscopically smooth surfaces, and which have been shown to dominate adhesion [33, 34]. Hence, they are expected to affect the adhesive friction. In fact, surface roughness was considered in Ref. [35] as a factor that can affect the stiffness of the interface. It is noteworthy that, even if at present, roughness might not be observable in contact area measurements. For instance, in Ref. [5], the pixel size was $25 \mu \mathrm{m}$, which would render any rough feature with wavelengths smaller than that unnoticeable.

\section{Conclusions}

A model that can describe the contact response of adhesive bodies under mixed-mode loading was presented herein. This model was applied to investigate tangentially loaded cylinder-on-flat contacts under adhesive conditions. A Maugis-like parameter, $\lambda_{t}$, was defined in the tangential direction and varied to change the relative stiffness of the body and interface. When $\lambda_{\mathrm{t}}$ is high, the interface is stiff as compared with the body, and a pressure spike is formed at the edge of the contact, similar to that observed in the adhesive normal pressure. In this case, the transition toward gross sliding occurred unstably with negligible local slip prior to the transition. When $\lambda_{\mathrm{t}}$ is low, the interfaces are compliant as compared with the body. This resulted in significant displacements at the interface prior to gross sliding and an almost constant tangential pressure throughout the entire contact area.

To validate the test model, the results yielded by it were compared qualitatively with experimental data 
in the literature. It was found that the model can qualitatively replicate the experimental observation, i.e., the contact area decreased according to a power law with an exponent of approximately 2 . In addition, a transition to gross sliding, in which the friction force and contact area decreased abruptly, was observed and is consistent with the experimental observations, although the transition was faster in our model. In general, the best agreement with the experimental observations was obtained when $\lambda_{\mathrm{t}} \approx 1$.

The model was compared with analytical models based on the LEFM to identify the similarities and differences. The main advantages of the proposed model compared with the analytical theories are that it can be used to investigate contacts with arbitrary geometries and various Maugis parameters, and that the values of difficult-to-measure parameters can be estimated.

\section{Acknowledgements}

This work is funded by the European Research Council (ERC) under the European Union's Horizon 2020 research and innovation programme (Grant No. 681813).

Open Access This article is licensed under a Creative Commons Attribution 4.0 International License, which permits use, sharing, adaptation, distribution and reproduction in any medium or format, as long as you give appropriate credit to the original author(s) and the source, provide a link to the Creative Commons licence, and indicate if changes were made.

The images or other third party material in this article are included in the article's Creative Commons licence, unless indicated otherwise in a credit line to the material. If material is not included in the article's Creative Commons licence and your intended use is not permitted by statutory regulation or exceeds the permitted use, you will need to obtain permission directly from the copyright holder.

To view a copy of this licence, visit http://creativecommons.org/licenses/by/4.0/.

\section{References}

[1] Sauer R A, Wriggers P. Formulation and analysis of a three- dimensional finite element implementation for adhesive contact at the nanoscale. Comput Methods Appl Mech Engrg 198(49-52): 3871-3883 (2009)

[2] Mergel J C, Sahli R, Scheibert J, Sauer R A. Continuum contact models for coupled adhesion and friction. $J$ Adhesion 95(12): 1101-1133 (2019)

[3] Shen L L, Glassmaker N J, Jagota A, Hui C Y. Strongly enhanced static friction using a film-terminated fibrillar interface. Soft Matter 4(3): 618 (2008)

[4] Waters J F, Guduru P R. Mode-mixity-dependent adhesive contact of a sphere on a plane surface. Proc $R$ Soc $A$ 466(2117): 1303-1325 (2010)

[5] Sahli R, Pallares G, Ducottet C, Ben Ali I E, Al Akhrass S, Guibert M, Scheibert J. Evolution of real contact area under shear and the value of static friction of soft materials. PNAS 115(3): 471-476 (2018)

[6] Sahli R, Pallares G, Papangelo A, Ciavarella M, Ducottet C, Ponthus N, Scheibert J. Shear-induced anisotropy in rough elastomer contact. Phys Rev Lett 122(21): 214301 (2019)

[7] Popov V L, Li Q, Lyashenko I A, Pohrt R. Adhesion and friction in hard and soft contacts: Theory and experiment. Friction 9(6): 1688-1706 (2021)

[8] Chateauminois A, Fretigny C, Olanier L. Friction and shear fracture of an adhesive contact under torsion. Phys Rev E 81(2): 026106 (2010)

[9] Prevost A, Scheibert J, Debrégeas G. Probing the micromechanics of a multi-contact interface at the onset of frictional sliding. Eur Phy J E, 36(2):1-12(2013)

[10] Savkoor A R, Briggs G A D. The effect of tangential force on the contact of elastic solids in adhesion. Proc $R$ Soc Lond A 356(1684): 103-114 (1977)

[11] Johnson K L. Adhesion and friction between a smooth elastic spherical asperity and a plane surface. Proc $R$ Soc Lond A 453(1956): 163-179 (1997)

[12] Papangelo A, Ciavarella M. On mixed-mode fracture mechanics models for contact area reduction under shear load in soft materials. J Mech Phys Solids 124: 159-171 (2019)

[13] Papangelo A, Scheibert J, Sahli R, Pallares G, Ciavarella M. Shear-induced contact area anisotropy explained by a fracture mechanics model. Phys Rev E 99(5): 053005 (2019)

[14] McMeeking R M, Ciavarella M, Cricrì G, Kim K S. The interaction of frictional slip and adhesion for a stiff sphere on a compliant substrate. J Appl Mech 87(3): 031016 (2020)

[15] Ciavarella M, Papangelo A. On the degree of irreversibility of friction in sheared soft adhesive contacts. Tribol Lett 68(3): 1-9 (2020)

[16] Wang J, Tiwari A, Sivebaek I M, Persson B N J. Sphere and cylinder contact mechanics during slip. J Mech Phy of Solids 143:104094 (2020)

[17] Peng B, Li Q Y, Feng X Q, Gao H J. Effect of shear stress on 
adhesive contact with a generalized Maugis-Dugdale cohesive zone model. J Mech Phys Solids 148: 104275 (2021)

[18] Mergel J C, Scheibert J, Sauer R A. Contact with coupled adhesion and friction: Computational framework, applications, and new insights. J Mech Phys Solids 146: 104194 (2021)

[19] McGarry J P, Ó Máirtín É, Parry G, Beltz G E. Potentialbased and non-potential-based cohesive zone formulations under mixed-mode separation and over-closure. Part I: Theoretical analysis. J Mech Phys Solids 63: 336-362 (2014)

[20] Salehani M K, Irani N, Nicola L. Modeling adhesive contacts under mixed-mode loading. J Mech Phy of Solids, 130: 320329 (2019)

[21] Khajeh Salehani M, Irani N, Müser M H, Nicola L. Modelling coupled normal and tangential tractions in adhesive contacts. Tribol Int 124: 93-101 (2018)

[22] Prodanov N, Dapp W B, Müser M H. On the contact area and mean gap of rough, elastic contacts: Dimensional analysis, numerical corrections, and reference data. Tribol Lett 53(2): 433-448 (2014)

[23] Xu X P, Needleman A. Void nucleation by inclusion debonding in a crystal matrix. Modelling Simul Mater Sci Eng 1(2): 111-132 (1993)

[24] Johnson K L, Greenwood J A. Maugis analysis of adhesive line contact. J Phys D: Appl Phys 41(19): 199802 (2008)

[25] Minn M, Sinha S K. The frictional behavior of UHMWPE films with different surface energies at low normal loads. Wear 268(7-8): 1030-1036 (2010)

[26] Vorvolakos K, Chaudhury M K. The effects of molecular weight and temperature on the kinetic friction of silicone rubbers. Langmuir 19(17): 6778-6787 (2003)

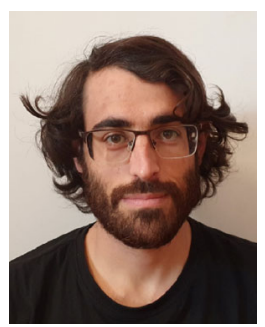

Francesc PÉREZ-RÀFOLS. He received his B.S. and M.S. degrees from Polytechnic University of Catalonia, Spain, and his Ph.D. degree in machine elements from

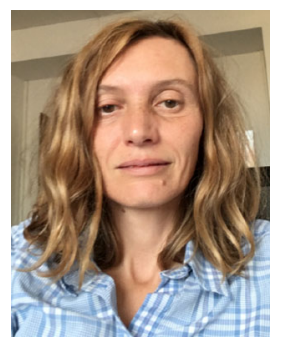

Lucia NICOLA. She received her M.S. degree in materials engineering from the university of Trento, Italy, and her Ph.D. degree in mathematics and physics from the university of Groningen, the
[27] Galliano A, Bistac S, Schultz J. Adhesion and friction of PDMS networks: Molecular weight effects. J Colloid Interface Sci 265(2): 372-379 (2003)

[28] Vaenkatesan V, Li Z L, Vellinga W P, de Jeu W H. Adhesion and friction behaviours of polydimethylsiloxane-A fresh perspective on JKR measurements. Polymer 47(25): 8317 8325 (2006)

[29] Rand C J, Crosby A J. Insight into the periodicity of Schallamach waves in soft material friction. Appl Phys Lett 89(26): 261907 (2006)

[30] Krick B A, Vail J R, Persson B N J, Sawyer W G. Optical in situ micro tribometer for analysis of real contact area for contact mechanics, adhesion, and sliding experiments. Tribol Lett 45(1): 185-194 (2012)

[31] Menga N, Carbone G, Dini D. Do uniform tangential interfacial stresses enhance adhesion? J Mech Phys Solids 112: 145-156 (2018)

[32] Hutchinson J W. Mixed mode fracture mechanics of interfaces. In Metal-Ceramic Interfaces, Acta-Scripta Metallurgica Proceeding Series, Volume 4. Rühle M, Evans A G, Ashby M F, et al., Eds. Oxford: Pergamon Press, 1990: 295-306.

[33] Pastewka L, Robbins M O. Contact between rough surfaces and a criterion for macroscopic adhesion. PNAS 111(9): 3298-3303 (2014)

[34] Greenwood J A. Reflections on and extensions of the fuller and tabor theory of rough surface adhesion. Tribol Lett 65(4): 1-12 (2017)

[35] Scheibert J, Prevost A, Frelat J, Rey P, Debrégeas G. Experimental evidence of non-Amontons behaviour at a multi-contact interface. Europhys Lett 83(3): 34003 (2008)

the University of Lulea, Sweden. Later, he was awarded a postdoc position at the University of Padova, Italy. Currently, he is a lecturer at Polytechnic University of Catalonia.

Netherlands. In 2017, she became a professor of computational materials science at Delft University of Technology, the Netherlands. Currently, she is a professor of metallurgy at the University of Padova, Italy. 